\title{
Avaliação do Desempenho Tribológico e Térmico de Materiais de Atrito Durante o Processo de Frenagem
}

\author{
Performance Evaluation Tribological and \\ Thermal Friction Materials During the \\ Braking Process
}

\author{
Rafael Lucas Machado Pinto ${ }^{1}$, Juan Carlos Horta Gutiérrez ${ }^{2}$, \\ Juan Carlos Campos Rubio ${ }^{2}$, Paulo Eustáquio de Faria ${ }^{2}$
}

\begin{abstract}
${ }^{1}$ Universidade Federal de Ouro Preto - Instituto de Ciências Exatas e Aplicadas - Rua 36, Número 115 - Bairro Loanda, João Monlevade, CEP: 35931-00. e-mail: rafaellucas@icea.ufop.br

${ }^{2}$ Universidade Federal de Minas Gerais - Av. Antônio Carlos, 6.627, CEP 31270-901 Belo Horizonte - MG. e-mail: horta@demec.ufmg.br, juan@demec.ufmg.br, paulofaria@eng-mec.dout.ufmg.br
\end{abstract}

\section{RESUMO}

Neste artigo, buscou-se verificar os parâmetros de operação de um sistema de freio a disco para motocicletas utilizando-se uma bancada de ensaios baseada no procedimento Krauss, descrita pela norma ABNT NBR $6143 / 1995$ [1]. Optou-se por conduzir um Experimento Fatorial $2^{3}$, em que os três fatores de controle considerados (tipo de disco de freio, tipo de pastilha e o valor da pressão de acionamento da manete de freio) foram alterados deliberadamente em dois níveis cada. Buscou-se analisar as propriedades dos materiais envolvidos novos e após um determinado tempo de uso, denominado materiais assentados. Para avaliar o desempenho do sistema de freios durante o processo de frenagem, analisaram-se quatro variáveis de resposta simultaneamente: temperatura final, coeficiente de atrito, desaceleração e momento da força de frenagem. Foram utilizadas técnicas de Planejamento de Experimentos Fatoriais para analisar os dados coletados. Como resultado, o desempenho tribológico mais satisfatório aconteceu para os materiais constituintes do par de atrito, disco e pastilhas, assentados, e um maior valor de pressão de acionamento da manete de freio. Já para a temperatura, o melhor resultados ocorreu para os materiais do par de atrito novo e um menor valor de pressão. Por fim, foi proposta uma técnica de otimização com o intuito de encontrar a melhor combinação dos níveis de cada fator que otimize o desempenho global das múltiplas variáveis resposta.

Palavras-chave: Material de atrito, Frenagem, Sistemas de Freio

\begin{abstract}
In this paper, we sought to verify the operating parameters of a disc brake system for motorcycles using a bench testing based on Krauss procedure described by the ABNT NBR 6143/1995 [1]. It was decided to conduct an experiment Factorial $2^{3}$, in which the three control factors considered (type of brake disc, type of chip and the value of the brake lever on working pressure) have changed on two levels each. It sought to analyze the properties of new materials involved, and after a certain time of use, called settlers materials. To evaluate the performance of the brake system during the braking process, we analyzed four response variables simultaneously: final temperature, coefficient of friction, deceleration time and braking force. Factorial Design of Experiments techniques were used to analyze the collected data. As a result, the most satisfactory tribological performance happened to the pair of materials constituting the friction disk and pads, setting, and an increased brake lever of the actuation pressure value. As for the temperature, best results occurred for the friction pair new materials with lower pressure. Finally, it proposed an optimization technique in order to find the best combination of the levels of each factor to optimize the overall performance of multivariable response
\end{abstract}

Keywords: Friction material, braking, brake systems. 


\section{INTRODUÇÃO}

De acordo com MAKRAHY et al. (2013) [13], sistemas de freio têm a finalidade de reduzir ou manter a velocidade de um veículo, levá-lo à imobilidade ou mantê-lo imóvel. Segundo SARIP (2011) [24], o princípio básico de um sistema de freio por atrito é converter a energia cinética do veículo em energia térmica pressionando dois materiais, um contra o outro, em contato para desenvolver atrito entre duas superfícies.

SISMAN (1936) [25] discutiu o desempenho de frenagem e testou métodos para determinação do desempenho do freio de um veículo, encontrando cinco principais fatores: coeficiente de atrito entre o pneu e a superfície da estrada, peso do veículo, esforço físico do condutor, atuação do sistema de frenagem e coeficiente de atrito do par de fricção. SANDERS et al. (2001) [23] ressalta que o coeficiente de atrito depende da velocidade de escorregamento, da pressão de contato e da temperatura na interface do par tribológico. Segundo YEVTUSHENKO e GRZES [28], a magnitude e flutuações no coeficiente de atrito durante uma frenagem dependem das propriedades mecânicas e termofísicas dos materiais de atrito, da velocidade, da carga aplicada, dos regimes de temperatura de contato bem como da natureza física e química do processo. $\mathrm{O}$ processo de frenagem resulta em contínuas e interdependentes mudanças também em relação a fatores externos, como velocidade, temperatura, umidade, propriedades das camadas sub-superficiais dos materiais e da intensidade de processo de contato na interface de atrito. NEIS (2012) [16] menciona que as variáveis que influenciam no desempenho (desgaste e atrito) dos materiais de fricção utilizados para frenagem são composição química e microestrutura tanto do disco quanto da pastilha, velocidade de deslizamento entre o par de atrito, pressão de contato, filme de friç̧ão, histórico térmico e de carga do material, temperatura e rugosidade do disco, além das condições ambientais de realização dos testes.

O desenvolvimento da composição ideal para materiais de fricção é uma das atividades mais complexas para fabricantes deste setor. OSTERMEYER (2001) [19] ressalta que pastilhas de freio geralmente são constituídas por mais de 20 ingredientes. Segundo OSTERMEYER e MULLER (2008) [21], pastilhas e lonas de freios devem apresentar baixo desgaste do material de atrito, manter o atrito estável à medida que há variação de temperatura, liberação mínima de resíduos e minimização da geração de ruído e vibrações. Além disso, BLAU (2001) [2] salienta que os materiais de fricção devem ser resistentes à corrosão e Lee e Kang (2007) [11] ressaltam sobre a importância de componentes veiculares recentemente desenvolvidos buscarem minimização do peso. De acordo com ERIKSON, BERGMAN e JACOBSOM (2002) [7], os materiais de fricção para utilização em freios normalmente são classificados em duas classes, semi-metálicos ou orgânicos. Já para os discos de freio, as características desejáveis são resistência térmica, mecânica, à corrosão e ao desgaste, e usinabilidade. De acordo com BREMBO (1997) [5], o material mais utilizado desde os anos 50 para produção de discos de freios veiculares é o ferro fundido cinzento, constituído com a fase perlítica e grafita lamelar.

Ensaios em laboratório são frequentemente empregados para caracterizar o desempenho de materiais de fricção. Segundo SANDERS et al. (2001) [23], máquinas de ensaios de frenagem possibilitam que as propriedades dos materiais de friç̧ão sejam medidas com maior precisão, minimizam o custo de máquina e permitem que o material de fricção seja ensaiado antes que o sistema de freio seja projetado. TIMTE (2000) [27] aponta a facilidade de instrumentação e controle mais preciso das variáveis envolvidas como vantagens ao utilizar a metodologia de avaliação de frenagens através de bancadas em laboratório.

ORTEL, NEUBURGUER e SABO (2010) [18] desenvolveram uma bancada de ensaios de frenagem para analisar o comportamento de freios de bicicletas. Com o desenvolvimento do equipamento, uma função histerese pode ser registrada, resultando em informações sobre a relação do nível de força aplicado pelas mãos do ciclista e a medida da força de frenagem que desacelera o sistema bicicleta-ciclista. Considerando-se um mesmo valor de força nas mãos aplicado pelo ciclista na manete de freio, os autores concluíram que sistemas com discos de freio de maior diâmetro apresentaram maiores valores de força de frenagem, o que significa maior efetividade na frenagem.

MAKRAHY et al. (2013 b) [14] compararam o desempenho de um novo sistema de freio a disco de cunha proposto com um sistema de freio a disco convencional utilizando um dinamômetro inercial. Verificou-se que o novo sistema de freio a disco cunha multiplicou em 3,7 vezes a força de frenagem sob as mesmas condições de operação. Este aumento foi atribuído ao efeito de auto-energizante do sistema de freio a disco cunha.

NEIS et al. (2015) [17], realizaram um estudo baseado na análise comparativa do desempenho de pastilhas freio aplicadas a um modelo de veículo, sendo uma original e 3 pastilhas de reposição adquiridas no mercado brasileiro. A performance das pastilhas foi avaliada em função do coeficiente de atrito, do desgaste 
e das características acústicas. Os ensaios de frenagem foram realizados utilizando um tribômetro de escala. Cinco parâmetros foram utilizados para caracterizar as pastilhas de freio: o coeficiente de atrito médio, a variação do coeficiente de atrito, a taxa percentual de fade, a taxa de desgaste específica, e a média geral de vibração. Para relacionar todos os parâmetros, os autores criaram um índice Q. A análise dos resultados mostrou que as pastilhas de freio originais apresentaram o maior valor e a menor variação do coeficiente de atrito, bem como a melhor resistência ao fade.

A ferramenta DOE (Design of Experiments), segundo Montgomery (2012) [15], é uma técnica estatística para planejar, conduzir e analisar os resultados de um experimento, a fim de validar uma determinada proposição para a solução de um problema. O presente trabalho aplicou a metodologia de planejamento de experimentos fatoriais Completo $2^{3} \mathrm{em}$ um banco de ensaios através do procedimento Krauss, decrito pela norma ABNT NBR 6143/1995 [1], responsável em analisar as condições das pastilhas de freio a disco através da determinação do coeficiente de atrito e desgaste através da bancada de ensaios Krauss. Três variáveis de controle sofreram alterações intencionais para avaliar o desempenho do sistema de freio a disco quanto a quatro variáveis de resposta. Por fim, foram propostos 4 cenário de simulação, utilizando-se uma técnica de otimização global, para encontrar os níveis ideais de cada fator que maximiza a performance global da frenagem.

\section{MATERIAIS E MÉTODOS}

\subsection{Descrição da máquina de ensaio de frenagem}

Optou-se por equipar a bancada de ensaios com um sistema de freio de motocicleta, pois os parâmetros produzidos por esta máquina para simular a frenagem, como torque e potência no eixo que contém o disco de freio, serem mais compatíveis com um veículo de menor porte. O sistema de freio utilizado corresponde ao de uma motocicleta com as seguintes características: motor de 4 tempos, refrigerado a ar, 125 cc de cilindradas, potência de $8,68 \mathrm{kw}$ a $6800 \mathrm{rpm}$, torque de 8 N.m a $6000 \mathrm{rpm}$.

A bancada de ensaios foi modificada para atender aos requisitos da norma ABNT NBR 6143/1995[1]. Para garantir uma rotação no eixo que contém o disco de 660rpm +/- 10rpm, requisito da norma, foi utilizado um sistema de transmissão de torque e potência por correias e polias. Para a obtenção do momento da força de frenagem, foi fabricado um dinamômetro, ilustrado pela Figura 1, baseado no princípio de funcionamento de uma alavanca. O caliper, ou pinça de freio, foi montado junto a esta alavanca, que possui uma célula de carga. Ao acionar o freio, a força de atrito atua em sentido contrário ao movimento de rotação do disco de freio. Essa força de atrito é tangencial em relação à força normal de contato da pastilha de freio com o disco e tende a promover o giro da alavanca. Adotando-se $F_{t}$ como a força tangencial que atua sobre o ponto de tomada de medição, em [N] e $r_{a}$ o comprimento da alavanca, em [m], torna-se possível calcular o momento da força de frenagem, em [N.m], através da relação expressa pela Equação 1:

$$
M_{t}=F_{t} \cdot r_{a}
$$

Para a obtenção da pressão hidráulica, foi construído um medidor de força hidráulica, representado pela Figura 2, utilizando-se um pequeno cilindro, um pistão e um retentor de vedação. Ao acionar o freio, a pressão na linha hidráulica atua promovendo o deslocamento do pistão. Através de uma célula de carga adaptada na extremidade livre do pistão, foi possível obter a força $F_{h}$, em [N], que o fluido pressurizado exerce na área do pistão, $A_{p}$ em $\left[\mathrm{m}^{2}\right]$.

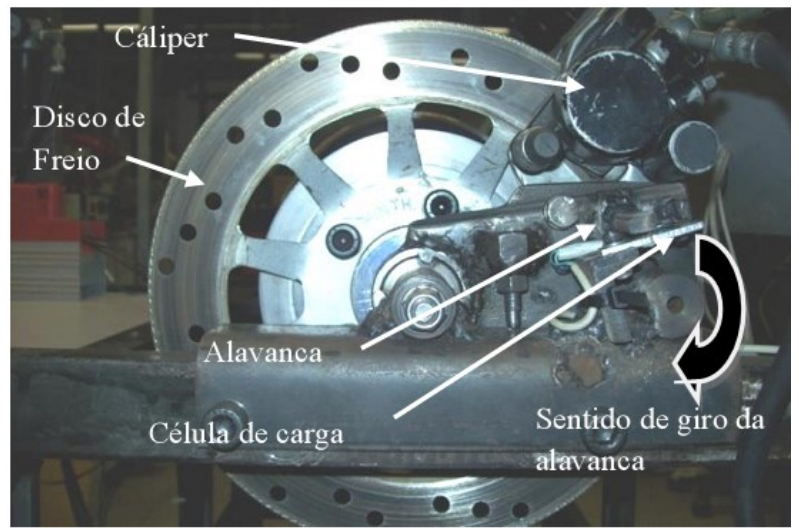

Figura 1: Dispositivo para tomada da força tangencial.

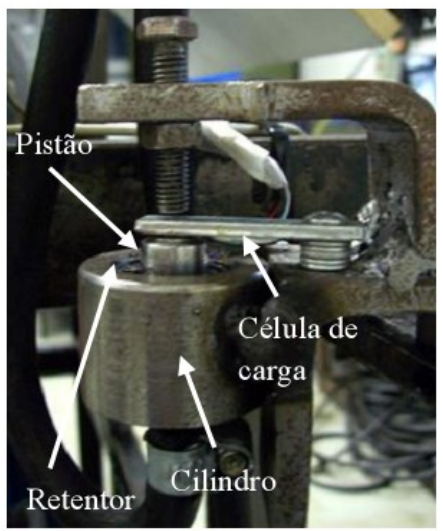

Figura 2: Dispositivo para tomada da força hidráulica. 
Para monitorar a velocidade de rotação do disco, foi utilizado um tacogerador eletrônico da marca Cycle Computer. A temperatura do disco foi obtida por meio de um pirômetro digital com sistema infravermelho de captura da marca Infrared Thermometer. As medições foram feitas na região do disco de freio imediatamente após a área de contato da pastilha.

Segundo HOFFMANN et al. (2006) [8], a força na manete de freio é um parâmetro relevante durante o estudo de frenagem, pois freios que exigem menor força das mãos, e que garantam a desaceleração desejada da motocicleta, aumentam o conforto proporcionado deste veículo ao condutor. O controle da força de atuação foi realizado através do controle da pressão da linha pneumática, uma vez que a manete de freio é acionada por um cilindro pneumático modelo DGS-25-80, marca Festo Pneumatic. O acionamento do cilindro é realizado através de uma válvula solenoide 5x2 vias, marca Bel Air. Para realizar a medição desta pressão pneumática, utilizou-se um manômetro com purgador da marca Werk Schott. A Figura 3 ilustra o diagrama esquemático do banco de ensaios montado neste trabalho.

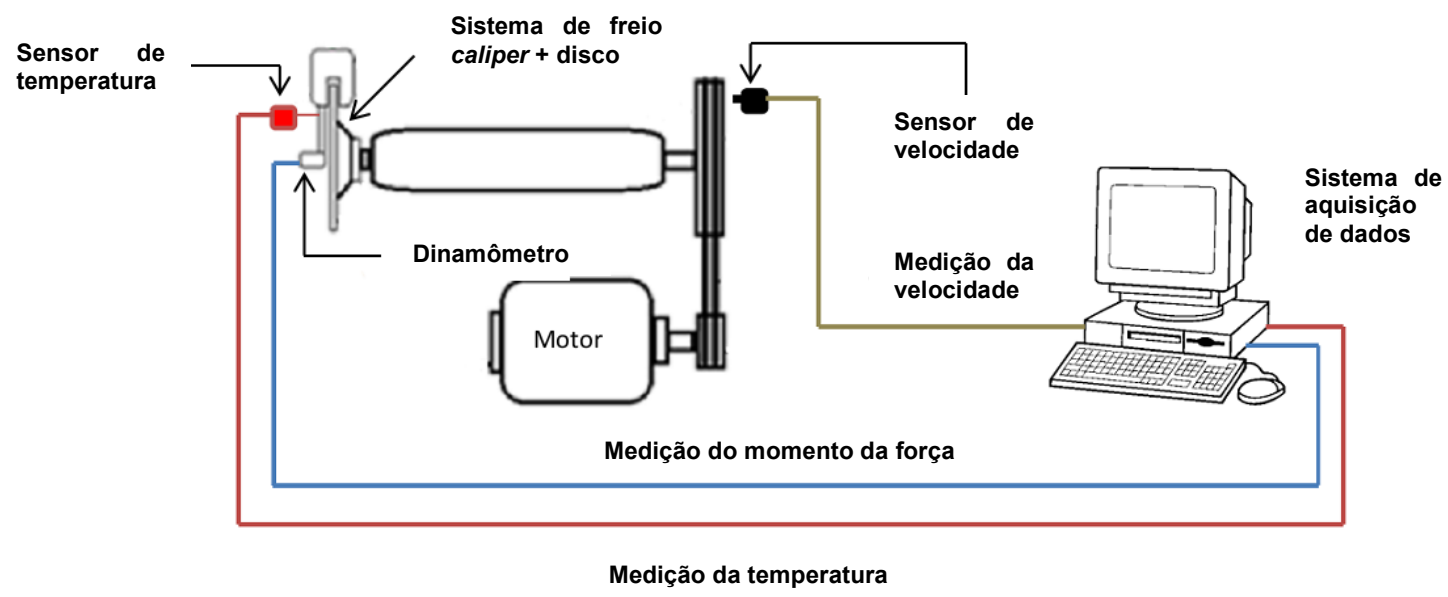

Figura 3: Representação esquemática do banco de ensaios.

\subsection{Cálculo do Coeficiente de atrito}

Uma das variáveis respostas analisadas como resultado dos ensaios é o coeficiente de atrito entre o par de atrito pastilha-disco. Adotando-se $M_{t}$ como o momento da força de frenagem, em [N.m], e $r_{e}$ como o raio efetivo do disco, em [m], a norma ABNT NBR 6143/1993[1] propõe o cálculo do coeficiente de atrito através da Equação 2:

$$
\mu=M_{t} /\left(2 . r_{e} \cdot A_{p} \cdot P_{h}\right)
$$

\subsection{Procedimento de Ensaio}

Os ensaios foram realizados utilizando-se 2 tipos diferentes de pastilhas - um par de pastilhas assentadas, que foi utilizado em uma moto similar, e um par de pastilhas nova. Foram utilizados dois tipos de discos de freio - um disco de freio novo e um em condições normais de uso, dito disco assentado. Utilizou-se também duas pressões pneumáticas diferentes para acionamento da manete de freio: 0,10 $\mathrm{MPa}$ e 0,15 $\mathrm{MPa}$, que geram pressões hidráulicas médias de acionamento do êmbolo do cilindro de freio de , respectivamente, 0,24 MPa e 0,47 MPa. A Tabela 1 apresenta a descrição de cada fator de controle manipulado em dois níveis no experimento.

Tabela 1: Níveis dos fatores Controláveis.

\begin{tabular}{l|c|c}
\hline \multicolumn{1}{c|}{ Fatores Controláveis } & Nível -1 & Nível +1 \\
\hline & & \\
X1: Tipo de Disco & Assentado & Novo \\
X2: Tipo de Pastilha & Assentada & Nova \\
X3: Valor da Pressão & $0,10 \mathrm{MPa}$ & $0,15 \mathrm{MPa}$ \\
\hline
\end{tabular}


Ainda de acordo com a norma utilizada, o ensaio é composto por 10 ciclos, cada um constituído por 10 frenagens, totalizando 100 frenagens. Os três primeiros ciclos são conduzidos para garantir assentamento das superfícies de trabalho. Os ciclos 4 a 10 somente podem ser executados caso haja um mínimo de $80 \%$ de assentamento entre o par de atrito. Estes foram os sete ciclos escolhidos para se empregar e técnica DOE.

Foram analisadas 4 variáveis de resposta: temperatura final em cada frenagem, coeficiente de atrito, desaceleração do sistema durante a frenagem e momento da força de frenagem. Para a temperatura final, quanto menor o seu valor melhor, uma vez que o sistema de freio fica menos sujeito a perdas de eficiência devido a efeitos térmicos, como, por exemplo, o fade. Já para o coeficiente de atrito, desaceleração e momento da força de frenagem, quanto maiores forem os seus valores melhor, uma vez que proporcionam maior efetividade durante a frenagem.

Utilizou-se a metodologia estatística planejamento fatorial completo $2^{3}$ do software Minitab 16 para gerar o experimento e analisar os dados coletados. Foi proposto também uma metodologia de otimização global, utilizando a ferramenta Response Optimizer, para encontrar a melhor combinação dos níveis de cada fator que resulte em uma otimização global das múltiplas variáveis simultaneamente.

\section{RESULTADOS}

\subsection{Análise da variável resposta temperatura final}

A Figura 4 ilustra o gráfico de Efeitos Principais para a variável resposta temperatura final. Percebe-se que esta variável é minimizada quando os ensaios foram realizados utilizando-se disco de freio novo, a pastilha nova (menos significativo) e pressão de acionamento de $0,10 \mathrm{MPa}$. Considerando-se um nível de significância de 0,05 , foi possível verificar que as interações entre disco e pastilha, disco e pressão de acionamento e a interação simultânea dos três fatores não foram significativas. Utilizando os fatores mostrados na Tabela 1, que variam nos seus níveis -1 e +1 , o modelo de regressão para predizer o comportamento da Temperatura Final, obtido através do software Minitab 16 e representado pela Equação 3, foi capaz de explicar aproximadamente $74 \%$ da proporção da variabilidade na resposta da temperatura final $\left(\mathrm{R}^{2}=73,99 \%\right)$.

$$
Y 1=148,44-17,13 * \text { disco }-7,51 * \text { pastilha }+33,99 * \text { pressão }+11,51 * \text { pastilha } * \text { pressão }
$$

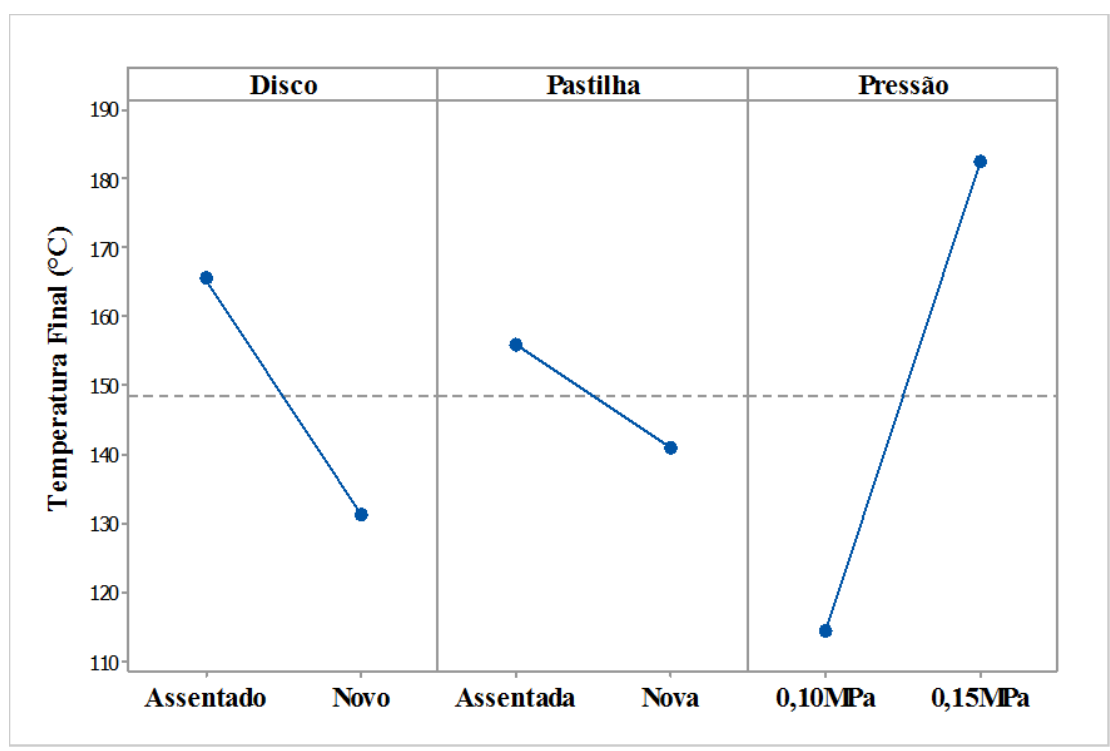

Figura 4: Gráfico de efeitos principais para a variável resposta temperatura final

Observando-se a Figura 5, que representa o gráfico de interação e os P-valores provenientes da análise de variância (ANOVA) para a variável resposta temperatura final, nota-se que a interação mais significativa ocorre entre os fatores pastilha e pressão de acionamento, conforme indicado na Equação 3 e através dos Pvalores indicados na Figura 5, pois é única que apresenta P-valor menor que a significância $\alpha=0,05$. A 
combinação dos níveis dos fatores que aperfeiçoa a temperatura final coincide com os resultados obtidos para o gráfico de efeitos principais.

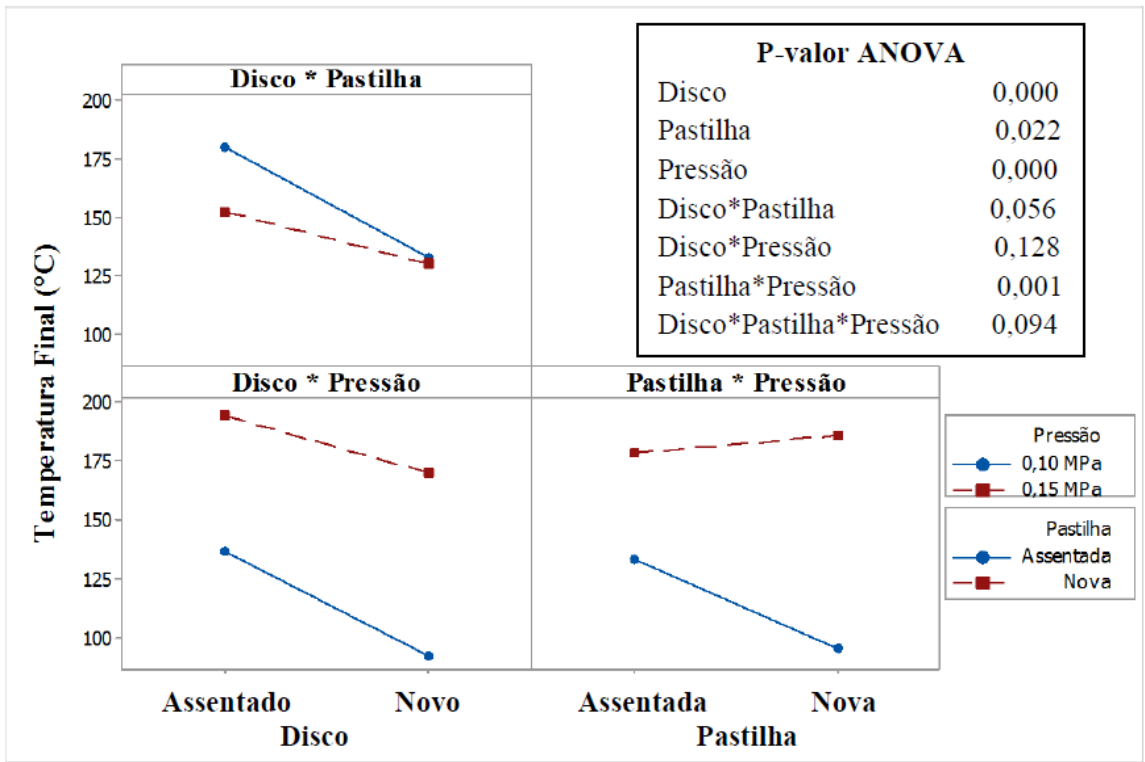

Figura 5: Gráfico de interação para a variável resposta temperatura final.

A Figura 6 ilustra a evolução da temperatura ao longo das frenagens 31 a 100 para os ensaios com pressão de acionamento da manete de freio de $0,10 \mathrm{MPa}$ e disco assentado, que apresentaram as menores temperaturas finais. As pastilhas assentadas alcançaram temperaturas finais mais elevadas se comparadas com as pastilhas novas. Isto decorre do maior grau de assentamento, promovendo uma maior área de contato entre pastilha e disco, em que os elementos abrasivos estão mais degastados. Desta forma, ocorre uma ação predominante do atrito entre as superfícies de trabalho e não a de cisalhamento por parte dos grãos abrasivos sobre o material do disco. Consequentemente, por apresentarem maior atrito, apresentaram maior aquecimento. Fenômeno semelhante pode ser observado na Figura 7, para os ensaios que apresentaram maiores valores para temperatura final (pressão $0,15 \mathrm{MPa}$, disco assentado).

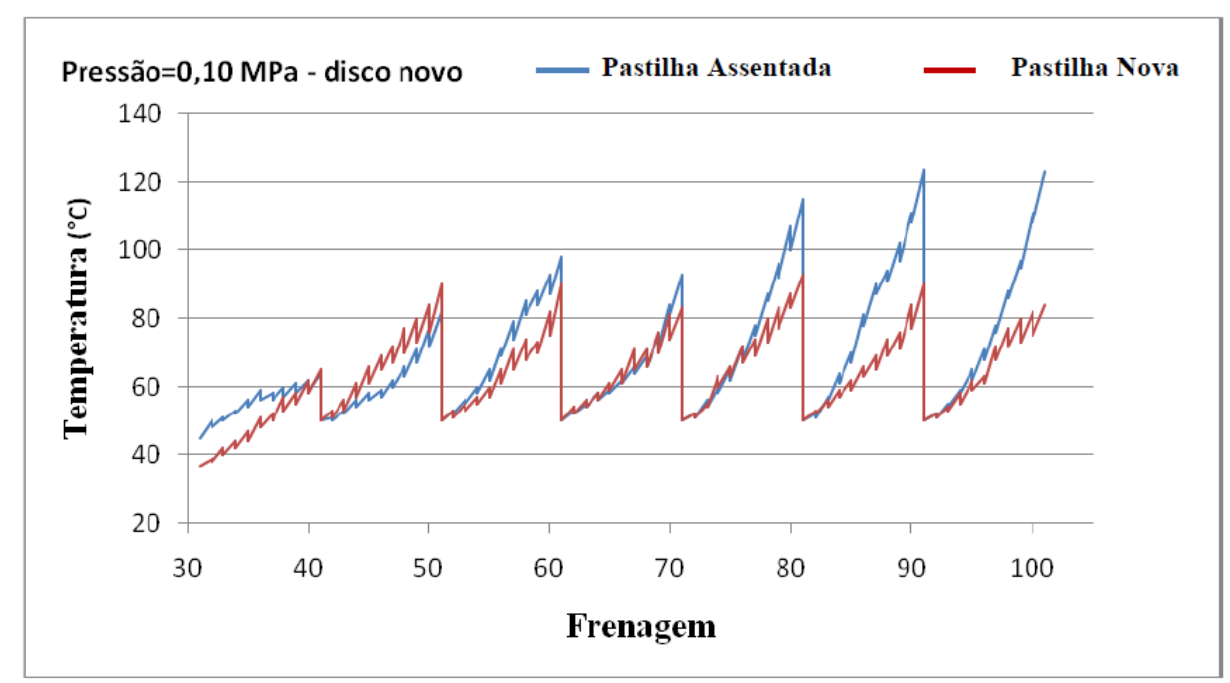

Figura 6: Gráfico da temperatura final para os ciclos 4 a 10 - Disco Novo.

Além disso, é possível perceber, conforme descrito por SONG e LEE (2009) [26], que a temperatura final de cada ciclo tende a ser crescente em concordância com o crescimento do número de frenagens. $\mathrm{O}$ stress térmico gerado no disco é obtido pela relação constituída pelo problema elástico com a expansão térmica. Daí o fato que a minimização da temperatura final otimiza o sistema de frenagem, pois minimizam os efeitos térmicos, como fade e instabilidade termo-elástica, que afetam o desempenho da frenagem. No 
presente estudo, restringiu-se a análise para os impactos do primeiro efeito térmico supracitado no sistema de frenagem considerado, sem a abordagem relativa ao segundo.

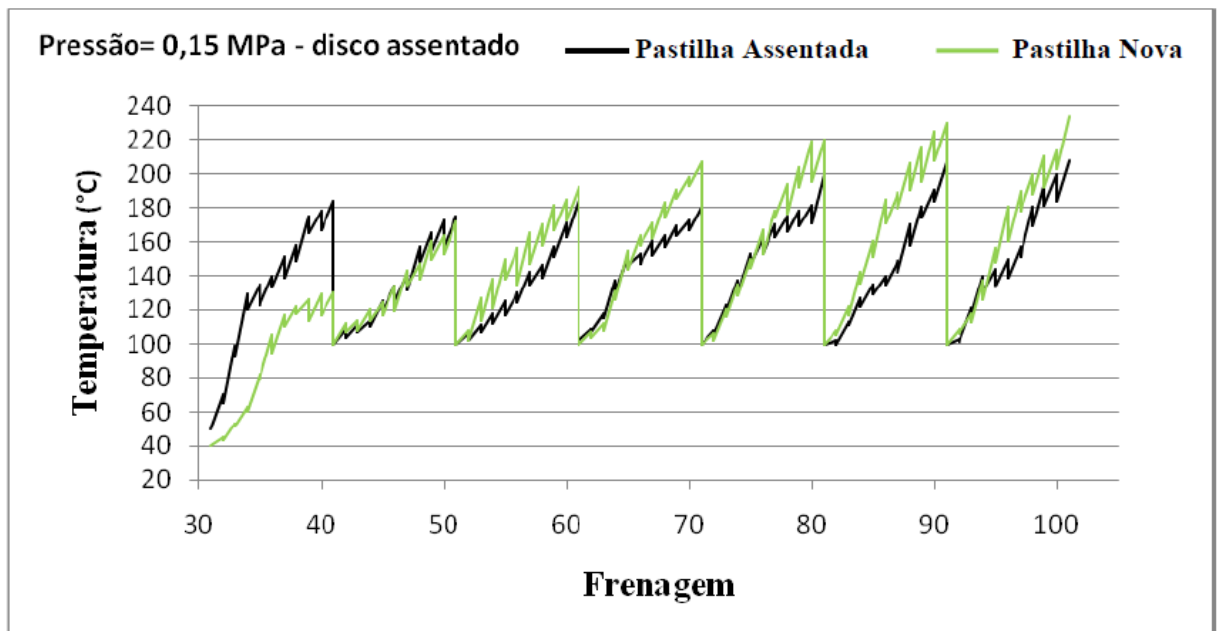

Figura 7: Gráfico da temperatura final para os ciclos 4 a 10 - assentado.

\subsection{Análise da Variável Resposta Coeficiente de Atrito}

A Figura 8 representa o gráfico de Efeitos Principais para a variável resposta coeficiente de atrito. Percebe-se que a mesma é maximizada quando o disco de freio é do tipo assentado, a pastilha é do tipo assentada e a pressão de acionamento é $0,15 \mathrm{MPa}$. Considerando-se um nível de significância de 0,05 , através da ANOVA é possível verificar que as interações entre disco e pastilha e a interação dos três fatores não são significativas. Estes resultados podem também ser observados através da Figura 9: as retas que representam a interação entre pastilha e disco são, aproximadamente, paralelas, o que significa não haver interação entre estes dois fatores. Já para a interação disco e pressão de acionamento e entre pastilha e pressão de acionamento, as retas não são paralelas, o que evidencia haver interação entre estes fatores ao nível de significância considerada. Tal interação entre estes fatores também pode ser observada através da análise do P-valor presente na Figura 9: ambas apresentam P-valor menor que a significância $\alpha=0,05$. O modelo de regressão obtido para predizer o comportamento do Coeficiente de Atrito, representado pela Equação 4, é capaz de explicar aproximadamente $81 \%$ da proporção da variabilidade na resposta $\left(\mathrm{R}^{2}=81,12 \%\right)$.

$Y 2=0,3118-0,03321 *$ disco $-0,02574 *$ pastilha $+0,00966^{*}$ pressão $+0,00862 *$ disco*pressão $+0,00723 *$ pastilha*pressão

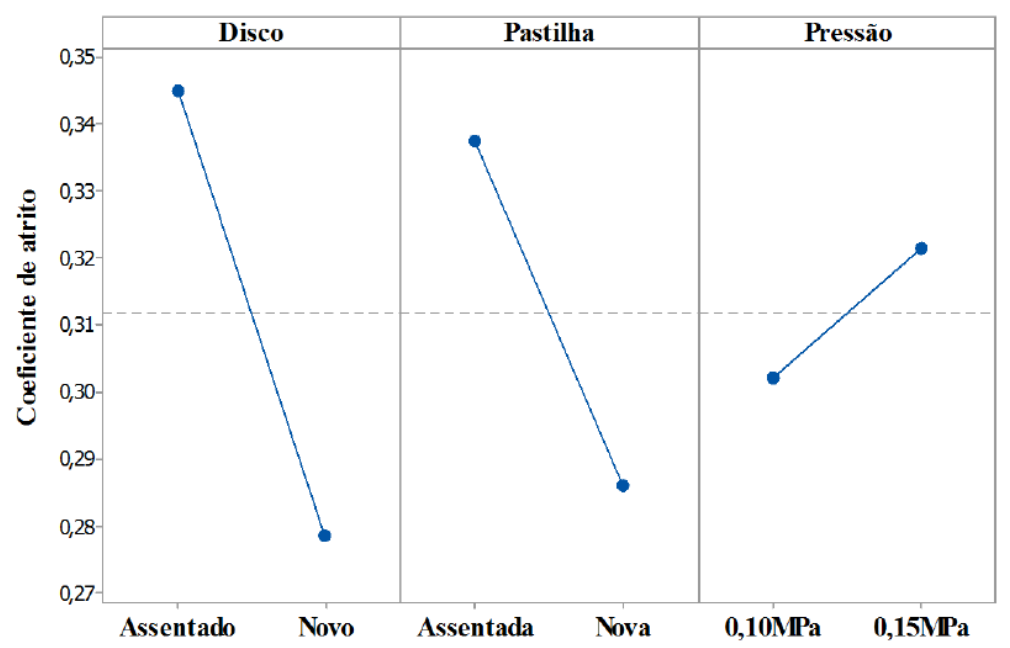

Figura 8: Gráfico de efeitos principais para a variável resposta coeficiente de atrito 


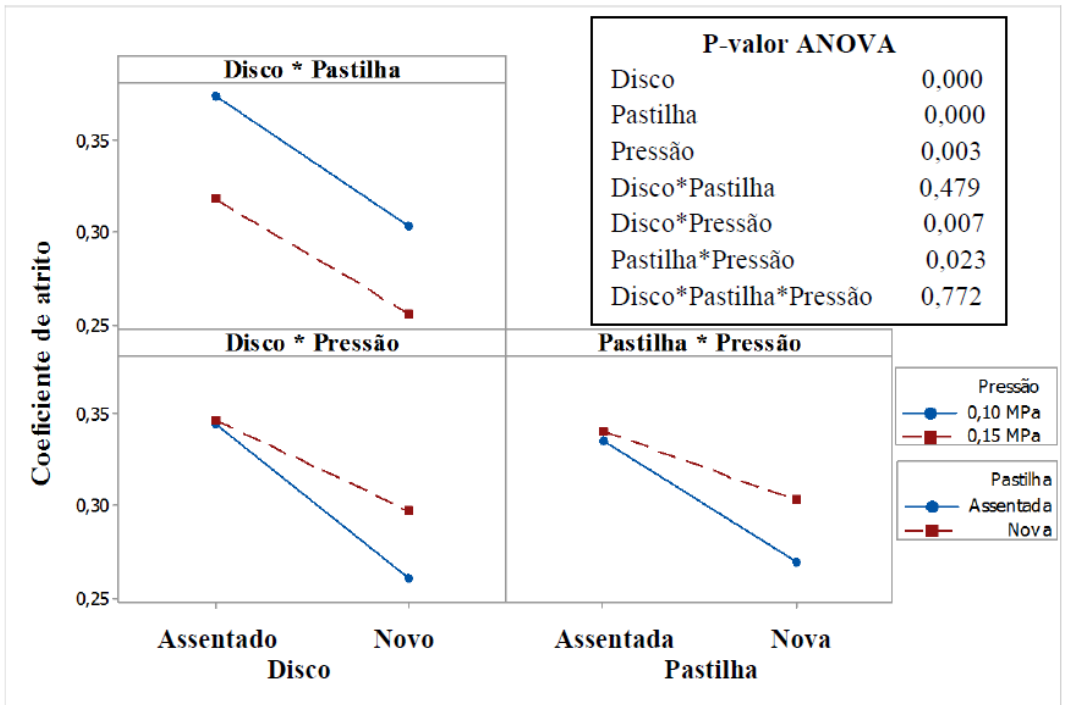

Figura 9: Gráfico de interação para a variável resposta coeficiente de atrito.

A Figura 10 ilustra o gráfico do coeficiente de atrito e da temperatura final para o último ciclo do ensaio que constituído por disco e pastilha assentada e pressão de 0,15 MPa, que maximizou o resultado da variável resposta coeficiente de atrito. É possível notar que a temperatura final de cada ciclo tende a ser crescente em concordância com o crescimento do número de frenagens e que os valores para o coeficiente de atrito apresentam também uma tendência crescente. Os valores obtidos para o coeficiente de atrito estão de acordo com os obtidos por NEIS (2012) [16] (0,30 a 0,45) e BODE e OSTERMEYER (2009) [4] (0,30 a $0,50)$ para esta faixa de temperatura. Comparando a Figura $10 \mathrm{com}$ a Figura 11, que representa o ensaio para o décimo ciclo de frenagem se utilizando disco assentado, pastilha nova e pressão de $0,15 \mathrm{MPa}$, nota-se que na última há redução no coeficiente de atrito para um número de frenagem maior que 6 e temperaturas na faixa de 50 a $120^{\circ} \mathrm{C}$.

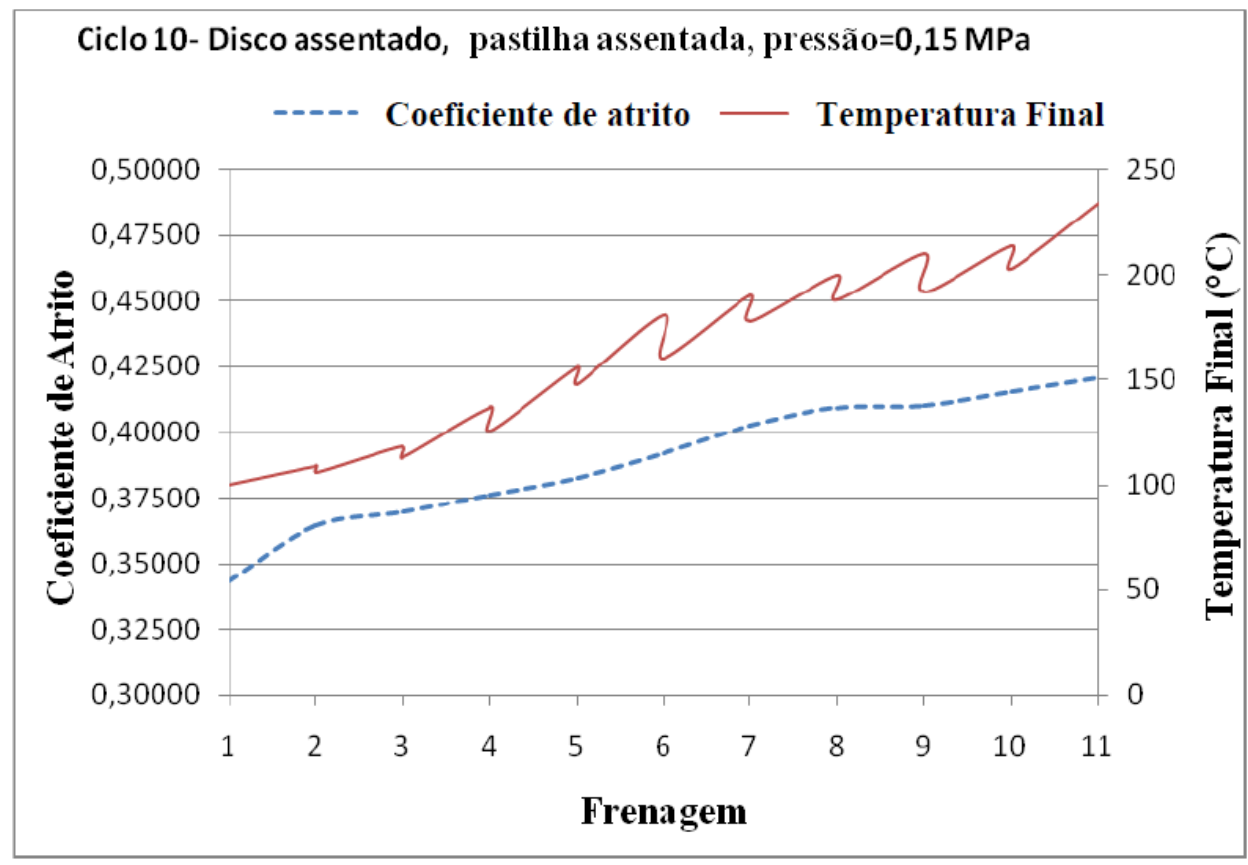

Figura 10: Coeficiente de atrito - pastilha assentada. 


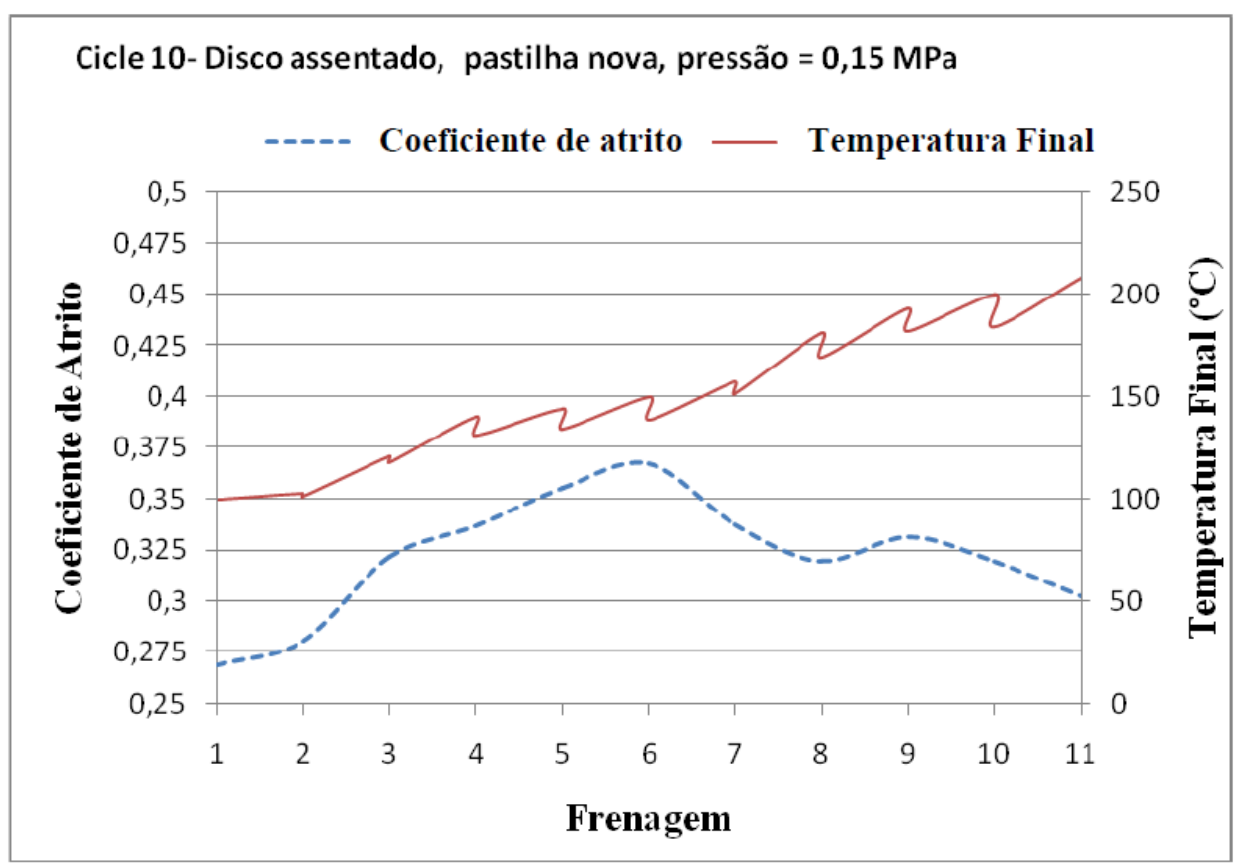

Figura 11: Coeficiente de atrito - pastilha nova.

A Tabela 2 representa, resumidamente, os níveis dos fatores que otimizam cada variável resposta separadamente. Foram comparados também os valores médios obtidos nos ensaios com os valores preditos através do modelo de regressão para os níveis ótimos. As análises das variáveis resposta desaceleração e momento da força de frenagem foram conduzidas de forma semelhante à da temperatura final e do coeficiente de atrito. Os ajustes dos níveis dos fatores que maximizam estas duas variáveis resposta foram os mesmos obtidos para o coeficiente de atrito, conforme esperado pelo literatura.

Tabela 2: Definição dos ajustes ótimos para cada variável resposta

\begin{tabular}{|c|c|c|c|c|}
\hline \multirow[b]{2}{*}{ Fatores Controláveis } & \multicolumn{4}{|c|}{ Variáveis resposta } \\
\hline & $\begin{array}{l}\text { Y1:Temperatura } \\
\text { Final }\end{array}$ & $\begin{array}{l}\text { Y2: Coeficiente } \\
\text { de atrito }\end{array}$ & Y3: Desaceleração & $\begin{array}{l}\text { Y4: Momento } \\
\text { da força }\end{array}$ \\
\hline Disco & Novo & Assentado & Assentado & Assentado \\
\hline Pastilha & Novo & Assentada & Assentada & Assentada \\
\hline Pressão & $0,10 \mathrm{MPa}$ & $0,15 \mathrm{MPa}$ & $0,15 \mathrm{MPa}$ & $0,15 \mathrm{MPa}$ \\
\hline $\begin{array}{l}\text { Percentual da } \\
\text { variabilidade explicada } \\
\text { pelo modelo }\left(\mathbf{R}^{2}\right)\end{array}$ & 73,99 & 81,12 & 64,49 & 94,3 \\
\hline Valor médio obtido & $85,0^{\circ} \mathrm{C}$ & 0,3677 & $9,743 \mathrm{~m} / \mathrm{s}^{2}$ & 27,57 N.m \\
\hline Valor predito & $78,3^{\circ} \mathrm{C}$ & 0,3646 & $9,341 \mathrm{~m} / \mathrm{s}^{2}$ & 27,57 N.m \\
\hline
\end{tabular}

\section{DISCUSSÃO}

Os resultados obtidos devem-se aos seguintes fatos:

Quanto ao tipo de disco de freio, o nível denominado assentado apresenta uma superfície mais polida que o disco novo, com menor presença de micro irregularidades do que em relação a um disco novo, aumentando, desta forma, a área de contato com as pastilhas. De acordo com BERGMAN et al. (1999) [3] a presença destes micro "buracos" sobre a superfície do disco diminui a área de contato, o que causa redução no coeficiente de atrito. Consequentemente, a temperatura final, a desaceleração e o momento da força aumentam utilizando-se um disco gasto, em função do aumento do coeficiente de atrito. 
Quanto ao tipo de pastilhas, as do tipo assentadas apresentam superfície de contato maior que pastilhas novas. Segundo HORTA GUTIÉRREZ et al. (2013) [N], pastilhas que apresentam elementos abrasivos mais degastados estão sujeitas a uma ação predominante do atrito entre os elementos e não a de cisalhamento por parte dos grãos abrasivos sobre o material do disco. Consequentemente, pastilhas assentadas aquecem mais, apresentam maiores coeficiente de atrito, desaceleração e momento da força. OSTEMEYER (2003) [20] afirma que o histórico de utilização da pastilha determina o estado atual da área de contato a nível microscópico e, por consequência, o valor atual do coeficiente de atrito.

Maiores valores de pressão pneumática promovem maiores valores de força de deslocamento do cilindro pneumático. Como o cilindro pneumático é o responsável por acionar a manete de freio, maiores valores de pressão hidráulica são fornecidos ao sistema hidráulico, o que gera uma maior força normal de deslocamento das pastilhas de freio contra a superfície do disco. Logo, maiores valores de pressão pneumática levam a uma maior pressão de contato do par pastilha-disco, maior coeficiente de atrito, maiores temperatura, desaceleração e momento da força de frenagem.

\subsection{Otimização Global}

Para realizar a otimização global deste sistema, foi utilizada a ferramenta Response Optimizer referente ao planejamento de experimentos fatoriais do software Minitab 16. Os critérios para encontrar as melhores configurações globais dos fatores são baseados na função desirability individuais $\left(\mathrm{d}_{\mathrm{i}}\right)$ e composta $\left(\mathrm{D}_{\mathrm{i}}\right)$. Este procedimento é equivalente à definição das funções objetivo em otimização. Diferentes configurações dos níveis dos fatores produzem um valor desta função entre 0 e 1 . As configurações ótimas dos fatores são as que maximizam a função objetivo. O uso desta ferramenta requer a definição do intervalo de especificação para cada variável resposta, a importância relativa e os pesos de cada variável resposta.

Para a determinação do intervalo de especificação aceitável para a temperatura, LIMPERT (2011) [12] ressalta que sistemas de freio quando alcançam temperaturas acima de $300^{\circ} \mathrm{C}$ ficam sujeitos ao efeito fade. Já RHEE e SCHWARTZ (1979) [22] destacam que até a temperatura de $220^{\circ} \mathrm{C}$ a taxa de desgaste dos materiais de fricção mantém-se razoavelmente constantes e, a partir de $220^{\circ} \mathrm{C}$, esta taxa aumenta exponencialmente. Logo, a especificação escolhida foi de 50 a $220^{\circ} \mathrm{C}$, com alvo de $100^{\circ} \mathrm{C}$.

NEIS (2012) [16] desenvolveu um tribômetro para caracterização do efeito isolado da temperatura sobre o atrito medido em materiais de frição utilizados em freios veiculares. Para temperaturas até $220^{\circ} \mathrm{C}$, utilizando-se pastilhas de freio metálicas e não metálicas com dois diferentes diâmetros, obteve valores para o coeficiente de atrito variando de 0,30 a 0,45. BODE e OSTEMEYER (2009) [4] avaliaram o efeito do histórico térmico e de carga sobre o atrito ao longo de frenagens veiculares utilizando-se um dinamômetro. Os valores obtidos para o coeficiente de atrito, que variou, aproximadamente, de 0,30 a 0,5 , ocorreu devido a mudanças do filme de fricção. Desta forma, foi definido que o intervalo de especificação para o coeficiente de atrito seria de 0,30 a 0,45 . Alvo: 0,40 .

Para a desaceleração, o valor sugerido pela Traffic Engineering Handbook (ITE, 2016 [10]) para uma desaceleração confortável é de aproximadamente $3,0 \mathrm{~m} / \mathrm{s}^{2}$. EL SHAWARBY et al. (2005) [6] analisaram a desaceleração de veículos que trafegavam a uma velocidade de $72 \mathrm{~km} / \mathrm{h}$. Os resultados indicaram uma média de $3,27 \mathrm{~m} / \mathrm{s}^{2}$. Para veículos que começaram o processo de frenagem distante da linha de retenção (> 111 metros), a média da desaceleração foi de $2,2 \mathrm{~m} / \mathrm{s}^{2}$, enquanto que nos casos em que o processo se iniciou próximo ( $<32$ metros), a média foi de $5,9 \mathrm{~m} / \mathrm{s}^{2}$. Gates et al. (2007) realizaram estudo utilizando-se filmagem em interseções, para veículos que transitavam com velocidade entre 40 e $80 \mathrm{~km} / \mathrm{h}$. O valor médio encontrado para o $85^{\circ}$ percentil da desaceleração para a amostra analisada foi de $3,7 \mathrm{~m} / \mathrm{s}^{2}$. Os veículos que estavam a uma velocidade superior a $64 \mathrm{~km} / \mathrm{h}$ apresentaram maiores taxas de desaceleração (atingindo valores acima de $5 \mathrm{~m} / \mathrm{s}^{2}$ ) comparados aos que estavam a velocidades menores (com valores de desaceleração da ordem de 2 $\mathrm{m} / \mathrm{s}^{2}$ ). Baseando-se nestas referências, o intervalo definido para a desaceleração foi de 2 a $6 \mathrm{~m} / \mathrm{s}^{2}$. Alvo: 3 $\mathrm{m} / \mathrm{s}^{2}$.

Já o intervalo de especificação para o Momento da Força de Frenagem foi definido com base nas observações do menor e do maior valores encontrados ao longo dos testes. Desta forma, foi definido de 25 a 30 N.m. Por se tratar de uma variável de otimização maximizadora, o valor alvo é de 30 N.m. Por se tratar de uma máquina de ensaio única, não foram encontrados valores de referência para a definição do momento de força compatíveis com esse projeto.

Foram propostos quatro cenários a fim de estudar o comportamento deste sistema baseado na função objetivo: cenário 1, onde as quatro variáveis de resposta apresentaram os mesmos níveis de importância relativa (IR) e mesmos pesos (P); cenário 2, situação em que os pesos foram maiores para que as variáveis resposta encontrassem-se dentro limites de especificações do que para os valores alvos, mantendo-se o 
mesmo de nível de importância relativa para todas as variáveis resposta; cenário 3, onde o coeficiente de atrito apresentou importância relativa dobrada em relação à demais variáveis resposta, mantendo-se os pesos iguais e o cenário 4, em que o coeficiente de atrito também apresentou importância relativa dobrada em relação à demais variáveis resposta, mas os pesos maiores para que as variáveis resposta encontrassem-se dentro dos limites de especificações. A Tabela 3 apresenta o resumo dos cenários de simulação e os resultados da função objetivo individual (d) e composta (D).

Tabela 3: Resultados referentes à função objetivo para os quatro cenários propostos

\begin{tabular}{|c|c|c|c|c|c|c|c|c|c|c|c|c|c|}
\hline \multirow{4}{*}{$\begin{array}{c}\text { Cenário } \\
\text { de } \\
\text { simulação }\end{array}$} & \multicolumn{12}{|c|}{ Variáveis Resposta } & \multirow{4}{*}{$\begin{array}{c}\text { Função } \\
\text { Objetivo } \\
\text { Composta } \\
\text { (Di) }\end{array}$} \\
\hline & \multirow{2}{*}{\multicolumn{3}{|c|}{$\begin{array}{c}\text { Temperatura Final } \\
\text { Objetivo: menor é } \\
\text { melhor } \\
\text { Especificação: } 50 \text { a } \\
220^{\circ} \mathrm{C}\end{array}$}} & \multirow{2}{*}{\multicolumn{3}{|c|}{$\begin{array}{c}\text { Coeficiente de Atrito } \\
\text { Objetivo: maior é } \\
\text { melhor } \\
\text { Especificação: } 0,30 \text { a } \\
0,45\end{array}$}} & \multirow{2}{*}{\multicolumn{3}{|c|}{$\begin{array}{c}\text { Desaceleração } \\
\text { Objetivo: maior é } \\
\text { melhor } \\
\text { Especificação: } 2 \text { a } \\
6 \mathrm{~m} / \mathrm{s}^{2}\end{array}$}} & \multirow{2}{*}{\multicolumn{3}{|c|}{$\begin{array}{l}\text { Momento da Força } \\
\text { Objetivo: maior é } \\
\text { melhor } \\
\text { Especificação: } 25 \text { a } \\
\text { 30N.m }\end{array}$}} & \\
\hline & & & & & & & & & & & & & \\
\hline & IR & $\mathrm{P}$ & $\mathrm{di}$ & IR & $\mathrm{P}$ & $\mathrm{di}$ & IR & $\mathrm{P}$ & $\mathrm{di}$ & $\mathrm{IR}$ & $\mathrm{P}$ & $\mathrm{di}$ & \\
\hline 1 & 1 & 1 & 0,18115 & 1 & 1 & 0,66308 & 1 & 1 & 1 & 1 & 1 & 0,41691 & 0,47306 \\
\hline 2 & 1 & 0,5 & 0,42562 & 1 & 0,5 & 0,81431 & 1 & 0,5 & 1 & 1 & 0,5 & 0,64569 & 0,68779 \\
\hline 3 & 1 & 1 & 0,18917 & 2 & 1 & 0,66683 & 1 & 1 & 1 & 1 & 1 & 0,39599 & 0,50642 \\
\hline 4 & 1 & 0,5 & 0,43494 & 2 & 0,5 & 0,81661 & 1 & 0,5 & 1 & 1 & 0,5 & 0,62928 & 0,71163 \\
\hline
\end{tabular}

Pela Análise dos resultados obtidos, percebe-se que os cenários 2 e 4, que dão maior peso para que as variáveis resposta se encontrem dentro de um determinado intervalo, apresentaram maiores valores para a função objetivo composta (D) e individuais (d) para cada variável resposta se comparados aos respectivos cenários que estabelecem o mesmo peso ao valor alvo e intervalo. O melhor resultado para a função objetivo composta foi obtido para o cenário 4 , cujo valor foi $\mathrm{D}_{4}=0,71163$. Analisando-se este cenário ótimo, é possível perceber que ele maximiza o valor da função objetivo Individual para a temperatura final e coeficiente de Atrito. Para a desaceleração, o cenário 4 apresentou desempenho igual aos outros cenários. Já para o momento da força de frenagem, o cenário 4 apresentou o segundo melhor resultado. $\mathrm{O}$ cenário ótimo apresentou a seguinte configuração quanto aos níveis dos fatores:

- Disco de freio: nível -1, que representa disco de freio assentado.

- Pastilha: nível ótimo -1, que equivalente à pastilha assentada.

- Pressão de acionamento: Considerando-se uma escala contínua, o resultado ótimo obtido, nível 0,6566 , representa uma pressão de $0,14 \mathrm{MPa}$.

\section{CONCLUSÕES}

O presente trabalho desenvolveu um banco de ensaios que permitiu analisar variáveis que influenciam um sistema de frenagem veicular. Este banco de ensaios foi equipado com um sistema de freios de uma motocicleta de 125 cilindradas e utilizou-se um planejamento de experimentos fatorial completo $2^{3}$ para conduzir os experimentos e analisar os dados coletados. Três variáveis de controle foram manipuladas em dois níveis distintos, e o desempenho do sistema de frenagem foi avaliado em relação a quatro variáveis de resposta.

Os resultados obtidos para cada variável resposta coincidem com os resultados encontrados na literatura. Para a variável resposta temperatura final, cujo objetivo é a minimização do seu valor, a combinação dos níveis das variáveis de entrada que otimizaram o desempenho do sistema de frenagem foi disco novo, pastilha nova e pressão de acionamento igual a $0,10 \mathrm{MPa}$. Já Para as variáveis de resposta coeficiente de atrito, desaceleração e momento da força de frenagem, cujos objetivos são a maximização dos seus valores, a combinação dos níveis das variáveis de entrada que otimizaram o desempenho do sistema de frenagem é disco novo, pastilha nova e pressão de acionamento igual a $0,15 \mathrm{MPa}$.

Por fim, foi apresentado um método de otimização global, propondo-se quatro cenários de simulação. $\mathrm{O}$ cenário 4 apresentou melhor performance em termos da maximização da função objetivo. Os resultados para este cenário ótimo são condizentes, tenho em vista que são muito próximos dos resultados ótimos de três das quatro variáveis resposta. 


\section{BIBLIOGRAFIA}

[1] ASSOCIAÇÃO BRASILEIRA DE NORMAS TÉCNICAS, Rio de Janeiro NBR 6143:1995 - Pastilha do Freio a disco - Determinação do atrito e desgaste através da bancada de ensaio Krauss.

[2] BLAU, P.J., Compositions, Functions and Testing of Friction Brake Materials and their Additives, Technical report, ORBL/TM-2001/64, Oak Ridge National Laboratory, 2001

[3] BERGMAN, F., ERIKSSON, M., JACOBSON, S., "Influence of Disc Topography on Generation of Brake Squeal”, Wear, v. 225-229, Part 1, pp. 621-628, 1999.

[4] BODE, K. H., OSTEMEYER, G. P., "Simulations and Tests of Innovative Friction Laws in Brake Systems", TechnischeUniversitatBraunschweig, Institute of Dynamics and Vibrations, v. 9, 1 ed., pp. 63-66, 2009.

[5] BREMBO, S.P.A.,The Brake Disc Manual, 1 ed., Manual, Curno, 128p, 1997

[6] EL SHAWARBY, I., RAKHA, H., INMAN, V. W., et al,"Evaluation of Driver Deceleration Behavior at Signalized Intersections".In: Transportation Research Record: Journal of the Transportation Research Board, n. 2018, Transportation Research Board of the National Academies, Washington, 2005

[7] ERIKSON, M., BERGMAN, F., JACOBSON, S. "On the Nature of Tribological Contact in Automotive Brakes", Wear, v. 252, ed. 1-2, p. 26-36, 2002.

[8] HOFFMAN, O., ECKERT, A., WOYWOD, J. "The Motorcycle Integral Brakesystem MIB: an advanced brake solution for high performance motorcycles", InternationaleMotorradkonferenz, Köln, v.6, n. 10, pp. 9 10,2006

[9] HORTA GUTIÉRREZ, J.C., ABREU, R.M, VASCONCELOS, I.R., et al., "Estudo Experimental do Desempenho de Sistema de Freio Automotivo", CIBIM, La Plata, 2013.

[10] ITE, Traffic Engineering Handbook, Institute of Transportation Engineers , 7 ed., 688p., Wiley, 2016.

[11] LEE, K. H., KANG, D. H., "Structural optimization of an automotive door using the kgiging interpolation method", Proc. IMechE, Part D: J. Automobile Eng, v. 221, n.12, pp. 1525-1534, 2007.

[12] LIMPERT, R., Brake Design and Safety, 3 ed., SAE, 2011.

[13] MAKRAHY, M. M., GHAZALY, N. M., ABD EL-GWWAD, K.A., et al., "Optmization of Operation Parameteres on a Novel Wedge Disc Brake by Taguchi Method",American Journal of Vehicle Design, v. 1, n. 2, pp. 30-35, 2013(a).

[14] MAKRAHY, M.M., NOUBY, M.G., ABD EL-GWWAD, K.A., et al., "A Preliminary Experimental Investigation of a New Wedge Disc Brake".Nouby M. Ghazaly et al Int. Journal of Engineering Research and Applications, v. 3, n. 6, pp 735-744, Nov-Dec 2013(b).

[15] MONTGOMERY, D.C., Introduction to Statistical Quality Control, 7 ed., John Wiley\& Sons, Inc., 2012.

[16] NEIS, P.D., Projeto e Construção de um Tribômetro com Controle Independente da Temperatura do Disco. Tese de D.Sc., Programa de Pós-Graduação em Engenharia Mecânica, Universidade Federal do Rio Grande do Sul, 2012.

[17] NEIS, P. D., FERREIRA, N. F., POLETTO, J. C., “A comparative analysis of original and aftermarkets brake pads sold in the Brazilian market", Journal of the Brazilian Society of Mechanical Sciences and Engineering, v. 1, p. 1-11, 2015.

[18] ORTEL, C., NEUBURGUER, H., SABO, A., "Construction of a test bench for bicycle rim and disc brakes”, Science Direct - Procedia Engineering, v.2, pp. 2943-2948, 2010.

[19] OSTERMEYER, G.P., "Friction and Wear of Brake Systems", Forschungim Ingenieurwesen, v. 66, pp. 267-272, 2001.

[20] OSTERMEYER, G.P., "On the Dynamics of the Friction Coefficient”, Wear, v. 254, 9 ed., p.852-858, 2003.

[21] OSTERMEYER, G.P., MULLER, M.,"New Insights into the Tribology of Brake Systems", Journal of Automotive Engineering, v. 222, 7ed., pp.1167-1200, 2008.

[22] RHEE, S.K., SCHWARTZ, H.W., "Test Methods for Automotive and Truck Friction Materials, American Society for Testing and Materials", American Society for Testing and Materials, paper 701, pp. 39-49, Philadelphia, 1979.

[23] SANDERS, P.G., DALKA, T.M., BASH, R.H., “A Reduced-Scale Brake Dynamometer for Friction 
Characterization", Tribology International, v. 34, pp. 609-615, 2001.

[24] SARIP, S.B., "Lightweight Friction Brakes for a Road Vehicle with Regenerative Braking", Thesis of D. Sc., School of Engineering Design and Technology of the University of Bradford - England, 2011.

[25] SISMAN, W., Brake performance, Institution of Automobile Engineers, 1936.

[26] SONG, B.C., LEE, K.H.,"Structural Optimization of a Circumferencial Friction Disk Brake with Consideration of Thermoelastic Instability", International Journal of Automotive Technology, v. 10, n. 3, pp. 321-328, 2009.

[27] TIMTE, M.A., "Compasion of Lining Output Generated Using AK-Master and FMVSS 105 Simulation Dynamometer Procedures", Society of Automotive Engineers, 2000.

[28] YEVTUSHENKO, A. A., GRZES, P., "Axisymmetric TEA of Temperature in a pad/disc brake system at temperature dependent coefficients of friction and wear", International Communications in Heat and Mass Transfer, v.39, pp.1045-1053, 2012. 